\title{
Atomic Scale Characterization of HgTe/CdTe Superlattices Using STEM Z- Contrast Imaging and VEELS
}

\author{
Lianfeng Fu, ${ }^{*}$ Nigel D. Browning, ${ }^{*, * *}$ Hye-son Jung, ${ }^{* * *}$ and Christoph Grein ${ }^{* * *}$ \\ * Department of Chemical Engineering and Materials Science, University of California at Davis, \\ Davis, CA 95616 USA \\ ${ }^{* *}$ National Center for Electron Microscopy, Lawrence Berkeley National Laboratory, Berkeley, CA \\ 94720 USA \\ *** EPIR Technologies Inc., 590 Territorial Drive, Bolingbrook, IL 60440 USA
}

There has been extensive studies performed in II-VI semiconductor materials for both the fundamental physics and their potential applications in light emitters, chemical sensors, UV coatings, medicines, etc [1]. Among the II-VI//II-VI quantum structure systems, HgTe/CdTe superlattices (SLs) are of particular interest in the field of long wavelength infrared detector due to their widely variable band-gaps [2]. It is known that the electrical and infrared detector properties of $\mathrm{HgTe} / \mathrm{CdTe} \mathrm{SLs}$ are strongly dependent on the growth quality of $\mathrm{HgTe} / \mathrm{CdTe}$ heterointerfaces and the band-gaps of SLs. Furthermore, the band-gaps of HgTe/CdTe SLs are related to the thickness of individual layers. Therefore, a complete characterization of $\mathrm{HgTe} / \mathrm{CdTe}$ heterointerfaces and an exact knowledge of the thickness variation in SLs and its relationship with the band-gaps are important to fully develop $\mathrm{HgTe} / \mathrm{CdTe}$ SLs for infrared detector devices.

In this study, we have used the scanning transmission electron microscopy (STEM) high angle annular-dark-field (HAADF) Z-contrast imaging and valence electron energy loss spectroscopy (VEELS) techniques to characterize the $\mathrm{HgTe} / \mathrm{CdTe}$ SL heterointerfaces. It is well known that Zcontrast imaging in STEM can easily identify the hetero-interfaces with different compositions [35]. The use of VEELS to determine the band gap and obtain other electronic information is also well established [6]. Therefore, the conjunction of Z-contrast imaging and VEELS analysis makes a very powerful technique for the interfacial characterization of SLs.

The $\mathrm{HgTe} / \mathrm{CdTe} \mathrm{SLs}$ were grown on $\mathrm{CdTe} / \mathrm{Si}(211) \mathrm{B}$ substrates using Molecular Beam Epitaxy (MBE) methods in EPIR Tech. STEM samples were prepared in the cross-sectional geometry using conventional cross-section sample preparation processes. The STEM Z-contrast imaging and VEELS experiments were performed in NCEM, LBNL using a $200 \mathrm{kV}$ Schottky field-emission gun (FEG) FEI Tecnai F20 UT STEM/TEM. The microscope is equipped with a double focusing Wien filter acting as a monochromator directly below the field-emission gun. This configuration in combination with a high-resolution Gatan Imaging Filter (GIF) system allows for a high energy resolution of $0.18 \mathrm{eV}$ or better, which makes accurate band gap measurement possible.

The results of STEM Z-contrast imaging of the HgTe/CdTe SLs show that the individual SL layers are well-defined and uniformly grown. The 200 periods of a $\mathrm{HgTe} / \mathrm{CdTe} \mathrm{SL}$ were identified to be epitaxially grown on a CdTe buffer layer with the orientation of $\mathrm{SL}_{(211)} / \mathrm{CdTe}_{(211)} / \mathrm{Si}_{(211)}$, as shown in Figure 1(a) and 1(b). The thickness of CdTe buffer layer was determined to be $7.0 \mu \mathrm{m}$ and that of $\mathrm{HgTe} / \mathrm{CdTe}$ SLs as $1.8 \mu \mathrm{m}$, which matches well with XRD data. The layer thicknesses $\mathrm{d}_{\mathrm{HgTe}} / \mathrm{d}_{\mathrm{CdTe}}$ of one sample near the substrate are determined to be $47 \pm 2 \AA / 41 \pm 2 \AA$. However, the thickness $\mathrm{d}_{\mathrm{HgTe}} / \mathrm{d}_{\mathrm{CdTe}}$ near the surface are slightly different, namely $51 \pm 2 \AA / 41 \pm 2 \AA$. VEELS analysis based 
on the $41 \AA \mathrm{CdTe}$ layer reveals that its band gap is about $1.65 \mathrm{eV}$, as shown in Figure 2, which agrees with band structure calculations. Band gap measurements of individual HgTe layer with different thickness will be discussed.

\section{References}

[1]. S.Rjujirawat et al., Appl. Phys. Lett. 74 (16) (1999) 2346.

[2]. D. Goren et al., J. Appl. Phys. 80 (9) (1996) 5083.

[3]. N.D. Browning et al., Nature 366 (6451) (1993) 143.

[4]. L.F. Fu et al., Appl. Phys. Lett. 87 (2005) 262904.

[5]. S. X. Zhang et al., Appl. Phys. Lett. 88 (2006) 012513.

[6]. R. Erni et al., Ultramicr. 104 (3-4) (2005) 176.

[7]. This work was performed at the NCEM, LBL and supported by DOE DE-AC02-05CH11231 and NSF DMR-0335364. This work was also supported by the AFR Lab at Wright-Patterson under FA8650-04-C-5423. Dr. Lyn Brown is gratefully acknowledged for managing this program.
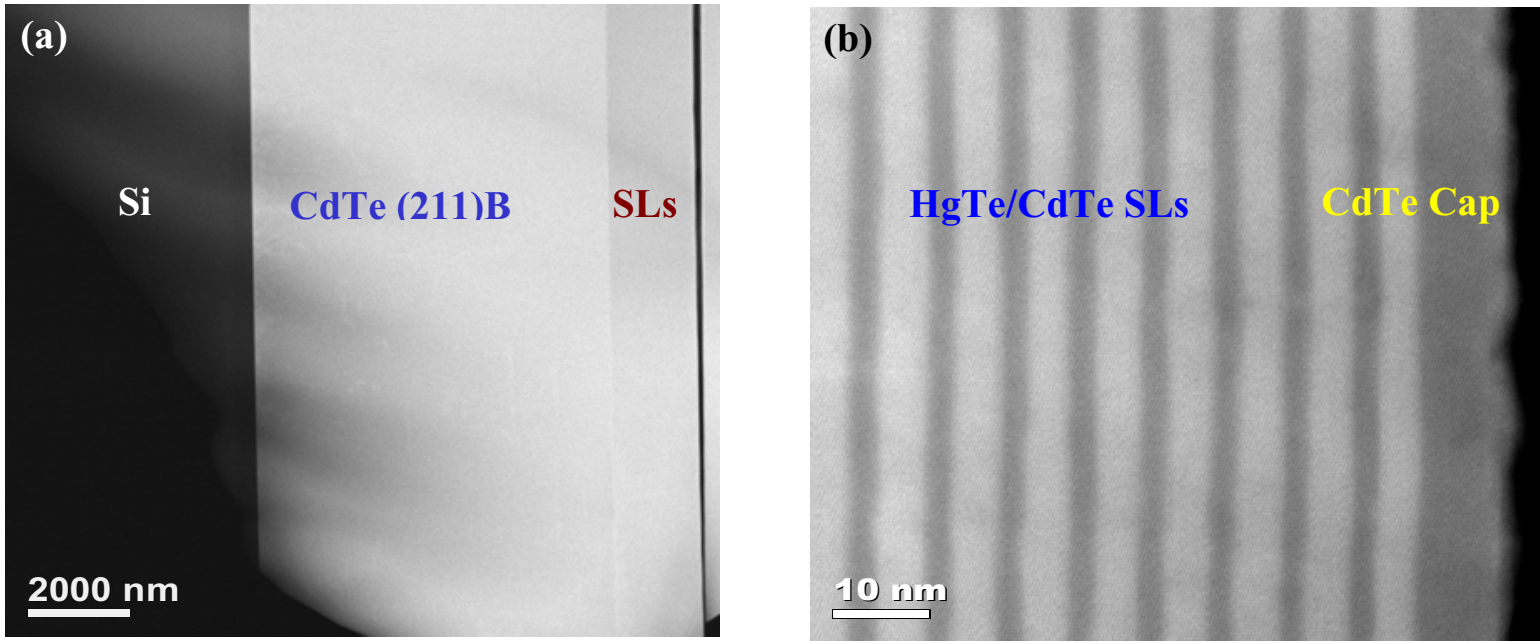

Fig. 1. (a) a cross-sectional STEM Z-contrast image of HgTe/CdTe SLs on a CdTe buffer layer and a Si substrate. (b) a high magnification Z-contrast image of $\mathrm{HgTe} / \mathrm{CdTe}$ SLs near surface, which shows the individual SL layers are well-defined.

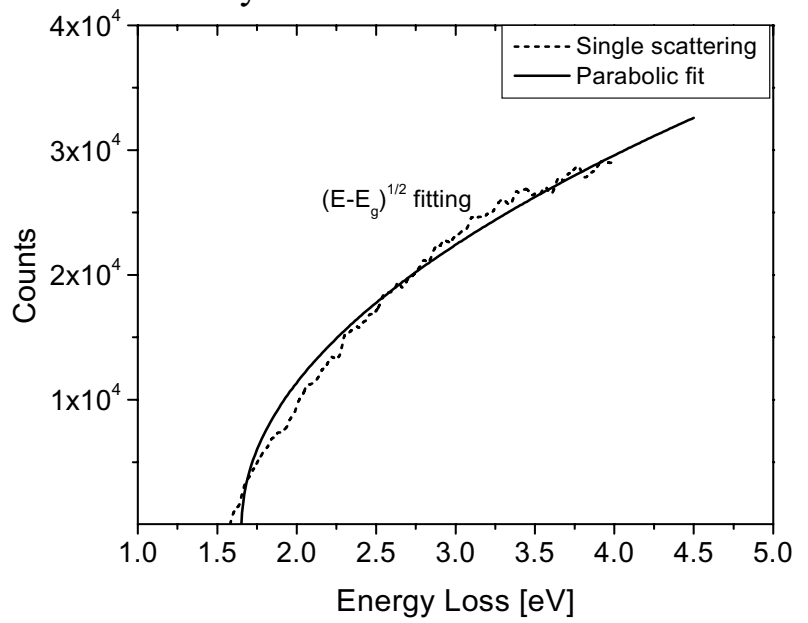

Fig. 2. Band gap region of the single scattering contribution of a CdTe layer revealing the band gap of $\sim 1.65 \mathrm{eV}$. The solid line shows the parabolic fitting used in our study. 